\title{
Transcriptome analysis of dog oral melanoma and its oncogenic analogy with human melanoma
}

\author{
MD. MAHFUZUR RAHMAN ${ }^{1,2}$, YU-CHANG LAI ${ }^{1}$, ALASMAUL HUSNA $^{1,2}$, \\ ${\text { HUI-WEN } \mathrm{CHEN}^{3} \text {, YUIKO TANAKA }}^{4}$, HIROAKI KAWAGUCHI ${ }^{5}$, HITOSHI HATAI ${ }^{6}$, \\ NORIAKI MIYOSHI $^{6}$, TAKAYUKI NAKAGAWA ${ }^{4}$, RYUJI FUKUSHIMA ${ }^{7}$ and NAOKI MIURA ${ }^{1-3}$
}

\author{
${ }^{1}$ Veterinary Teaching Hospital, Joint Faculty of Veterinary Medicine, Kagoshima University, Kagoshima 890-0065; \\ ${ }^{2}$ The United Graduate School of Veterinary Science, Yamaguchi University, Yamaguchi 753-8511; \\ ${ }^{3}$ Joint Graduate School of Veterinary Medicine, Kagoshima University, Kagoshima 890-0065; \\ ${ }^{4}$ Department of Veterinary Surgery, Graduate School of Agricultural and Life Sciences, The University of Tokyo, \\ Tokyo 113-8657; ${ }^{5}$ Hygiene and Health Promotion Medicine, Kagoshima University Graduate School of Medicine and \\ Dental Science, Kagoshima University; ${ }^{6}$ Department of Veterinary Histopathology, Joint Faculty of Veterinary Medicine, \\ Kagoshima University, Kagoshima 890-0065; ${ }^{7}$ Animal Medical Center, Tokyo University of Agriculture \\ and Technology, Tokyo 183-8538, Japan
}

Received May 4, 2019; Accepted September 30, 2019

DOI: $10.3892 / o r .2019 .7391$

\begin{abstract}
Dogs have been considered as an excellent immunocompetent model for human melanoma due to the same tumor location and the common clinical and pathological features with human melanoma. However, the differences in the melanoma transcriptome between the two species have not been yet fully determined. Considering the role of oncogenes in melanoma development, in this study, we first characterized the transcriptome in canine oral melanoma and then compared the transcriptome with that of human melanoma. The global transcriptome from 8 canine oral melanoma samples and 3 healthy oral tissues were compared by RNA-Seq followed by RT-qPCR validation. The results revealed 2,555 annotated differentially expressed genes, as well as 364 novel differentially expressed genes. Dog chromosomes 1 and 9 were enriched with downregulated and upregulated genes, respectively. Along with 10 significant transcription site binding motifs; the NF- $\mathrm{B}$ and ATF1 binding motifs were the most significant and 4 significant unknown motifs were indentified among the upregulated differentially expressed genes. Moreover, it was found that canine oral melanoma shared $>80 \%$ significant oncogenes (upregulated genes) with human melanoma, and JAK-STAT was the most common significant pathway between the species. The results identified a 429 gene signature in melanoma, which was up-regulated in both
\end{abstract}

Correspondence to: Dr Naoki Miura, Veterinary Teaching Hospital, Joint Faculty of Veterinary Medicine, Kagoshima University, 1-21-24 Korimoto, Kagoshima 890-0065, Japan E-mail: k9236024@kadai.jp

Key words: melanoma, transcriptome, analogy, dog, oncogenes species; these genes may be good candidates for therapeutic development. Furthermore, this study demonstrates that as regards oncogene expression, human melanoma contains an oncogene group that bears similarities with dog oral melanoma, which supports the use of dogs as a model for the development of novel therapeutics and experimental trials before human application.

\section{Introduction}

In recent years, dogs have been suggested as a model for several types of human cancer, including melanoma $(1,2)$. Melanoma is the most lethal skin cancer affecting humans. According to 'Cancer statistics, 2018' from the American Cancer Society, a total of 9,320 deaths were estimated in 2018, only in the US (3). However, the global incidence of melanoma is more of a concern (4). Cutaneous melanoma is the most common form of melanoma among individuals with fair skin, whereas non-cutaneous melanoma occurs in a greater proportion in populations of other ethnic groups $(4,5)$. Oral melanoma is the most common melanoma type among dogs and accounts for $7 \%$ of all malignant tumors in dogs (6). The median progression-free survival of dogs with oral melanoma is <200 days even following excision and DNA vaccination $(6,7)$.

It has recently been reported that human mucosal and dog oral melanoma bear more similar genetic alterations, such as copy number variations (CNVs), single nucleotide variations (SNVs) and mutations or deletions than human cutaneous melanoma (8). More similarities have also been observed in tumor location and histology with the mucosal than the cutaneous type $(2,8)$. Moreover, the genomic classification of cutaneous melanoma has revealed a subtype without mutation that exhibits increased aggressiveness, such as mucosal melanoma $(9,10)$. Due to these similarities between both species, dog oral melanoma has been suggested as a suitable model for 
both mucosal and triple wild-type human melanoma $(2,10,11)$. Several genetic mutations or loss of function events observed in human melanoma have also been identified in dog oral melanoma, such as $\mathrm{BRAF}^{\mathrm{V} 600 \mathrm{E}}$ (12), NRAS (Q61) mutation (11), loss function of phosphatase and tensin homolog (PTEN) (11) and c-KIT mutation and/or overexpression (13).

Genomic instability is a hallmark of cancer. Some aberrant genes promote cancer progression, while simultaneously inhibiting normal cellular process, whereas other deregulated genes occur as passenger alterations. The identification of specific cancer-causative genes may be effective for the development of therapeutic strategies against cancer. In this study, we used a novel technique to identify genes that are involved in melanoma development. We hypothesized that cancer-causing genes include orthologous genes that are altered within the same type of cancer among different species. Our hypothesis is an extension of cancer research that has been used for a number of years: Recurrent abnormalities among multiple cases are more likely to be causative factors than non-recurrent events. Our view was that recurrently aberrant orthologous genes in the same type of cancer between two related species are the main causative agents for disease progression. We extended our analysis between dogs and humans, which share ancestral DNA and have a similar incidence of melanoma $(2,14)$. This approach can better distinguish melanoma-causing genes from passenger aberrations, which may appear as a miscue in a single species investigation.

Previous reports have suggested dogs as a model for human melanoma. However, the genes and pathways involved in melanoma susceptibility have not yet been studied between species, at least to the best of our knowledge. In this study, we systematically analyzed and compared the canine and human melanoma transcriptome to address two objectives: To identify gene expression similarities between dog and human melanoma, and to examine common functional aspects of genes regulated during melanoma development between the species. We identified common differentially expressed genes (DEGs) between the two species and revealed causative or active genes involved in the pathogenesis of melanoma, which may further aid in the development of more effective therapeutic approaches for melanoma in both species.

\section{Materials and methods}

Tissue samples. Dog oral melanoma tissue samples $(\mathrm{n}=17)$ were obtained following surgical resection (as a primary treatment for the melanoma patient) at the Kagoshima University veterinary teaching hospital. The patient's owners were informed prior to sample collection. Confirmed diagnosis was affirmed by the hospital. Tissue samples were maintained immediately in RNAlater $^{\mathrm{TM}}$ (Invitrogen; Thermo Fisher Scientific) following isolation and incubated overnight at $4{ }^{\circ} \mathrm{C}$ and then stored at $-80^{\circ} \mathrm{C}$ until further RNA extraction. Detailed information of the 17 samples is listed in Table SI. Control oral tissues were obtained following surgical resection from healthy dogs $(n=12)$ during routine anatomical practical training classes from the Kagoshima University shed. The site (oral melanoma or healthy oral tissue) and general surgical procedure for sample collection was the same between the healthy dogs and those with melanoma. Anesthesia was performed and maintained accordingly during the surgical procedure [pre-administration: Atropine sulfate $20 \mu \mathrm{g} / \mathrm{kg}$ (i.v.), Robenacoxib $2 \mathrm{mg} / \mathrm{kg}$ (i.v.); induction: Propofol $\sim 5 \mathrm{mg} / \mathrm{kg}$ (i.v.); Maintenance: Sevoflurane $0.5-5 \%$ (inhalation)]. The anesthesia regimen was according to the American Animal Hospital Association (AAHA) guidelines (15). Palpebral and jaw reflexes were used to confirm that the animals were fully anesthetized. Other monitoring parameters, such as temperature, heart and respiratory rate, blood pressure, oxygen saturation, end tidal $\mathrm{CO}_{2}$, etc. were continuously checked during this period. Animals were not euthanized as part of the current study. The study design and experimental protocols were approved by the university and the Kagoshima University veterinary teaching hospital ethics committee (KV0004)

RNA extraction and sequencing. The mirVana ${ }^{\mathrm{TM}}$ miRNA isolation kit (Thermo Fisher Scientific lnc.) was used to isolate total RNA from the tissues according to the manufacturer's protocol. RNA concentration was measured using a NanoDrop 2000c Spectrophotometer (Thermo Fisher Scientific lnc.). RNA quality and integrity was assessed using the Agilent 2100 Bioanalyzer (Agilent Technologies). The RNA integrity number (RIN) mean value for the tissue was 8.8 (range 7-10).

Following RNA isolation and quality assurance, small RNA libraries were prepared and sequenced by Hokkaido System Science Co., Ltd. The TruSeq RNA Sample Prep kit version 2 (Illumina) was used for library preparation. The low sample protocol was followed and input total RNA was $0.5 \mu \mathrm{g}$. Briefly, PolyA-containing mRNA was purified using oligo-dT-attached magnetic beads. mRNA was fragmented into small sections following purification under an elevated temperature $\left(94^{\circ} \mathrm{C}\right)$ using divalent cations. Fragmented mRNAs were copied into first-strand cDNA using reverse transcriptase with random primers. Second-strand cDNA synthesis was followed by DNA polymerase I and RNase $H$ treatment. cDNA fragments underwent end repair process, a single addition of ' $A$ ' base and then ligation of adapters. The final cDNA library was created through purification and enrichment with PCR process.

Bioinformatics analysis. For bioinformatics analysis, the below procedures and analyses were performed.

Reads processing and differential expression analysis. We received high quality reads from the sequencing facilities average Phred score $>36$. Sequencing data were imported into the CLC Bio Genomics Workbench (CLC Bio; Qiagen) as recommended by the manufacturer's manual (http://resources. qiagenbioinformatics.com). The normalization of reads, quality, ambiguity and adapter trimming or quality control was performed with the CLC Bio Genomics Workbench (versions 9 and 10). Paired end reads (100 bp) were further analyzed according to the RNA-seq analysis guide of the CLC Genomics Workbench. Default parameters were used during mapping and all other subsequent analysis. Briefly, during reads mapping to a genome, genome annotated with genes and transcripts were selected and a mRNA track, gene track and a genome track Canis familiris.canfam3.1 were used (16). Reference sequences were downloaded using the workbench downloading option. During counting, the reads for expression values and the intact pairs were counted, while the broken 
pairs were ignored. The expression value was calculated in total counts, unique counts, transcripts per million, and reads per kilobase of exon model per million mapped reads (RPKM) (17). Differential Expression for the RNA-Seq tool was used to perform the statistical differential expression test. This tool followed a multi-factorial statistics based on a negative binomial Generalized Linear Model. The Wald test was used for comparison between the groups. We set the criteria for differential expression genes as false discovery rate (FDR) $<0.05$, fold change $(\mathrm{FC})>2$ (both upregulated and downregulated), and maximum group mean $>5$ (RPKM).

Cross species analysis of DEGs. We downloaded 3 RNA-seq datasets from the GEO database: GSE71747 for the human melanoma tissue, GSE88741 for the human melanoma cell line and GSE29155 for human prostate cancer. The datasets included for the cross-species analysis are illustrated in Fig. S1. Data were downloaded directly to the genomic workbench and the above-mentioned procedures and criteria were followed to analyze the reads. Human ortholog genes were collected by the BioMart tool within Ensembl (18). Comparisons were drawn regarding the $\mathrm{FC}$ and with or without statistical significance of the ortholog genes between the species.

Gene ontology $(G O)$, pathways and transcription factor analysis. GO and transcription factor analysis was performed by the WebGestalt (WEB-based GEne SeT AnaLysis Toolkit) (19) following the gene set enrichment analysis (GSEA) method. For pathway analysis, we blended 2 methods from WebGestalt and Pathview (20). We performed GSEA using the WebGestalt and Generally Applicable Gene-Set Enrichment (GAGE) by Pathview according to their default settings. Finally significant (q value or FDR <0.05) pathways from the two methods were selected.

Network analysis. Common DEGs were uploaded to STRING (https://string-db.org/) to obtain the protein interaction network (21). The parameter for the confidence score was set to 7. Cytoscape 3.5.1 (https://cytoscape.org/) was used to analyze the network (22). Closed networks were considered during network construction both in STRING and Cytoscape. MCODE algorithm was used within the Cytoscape application for cluster network analysis.

$R T$ - $q P C R$. Total RNA (250 ng) was reverse transcribed into cDNA using the ReverTra Ace ${ }^{\circledR}$ qPCR RT Master Mix with gDNA Remover (Toyobo). RT-qPCR was performed using a TaqMan ${ }^{\circledR}$ Fast Advanced Master Mix kit (Thermo Fisher Scientific Inc.) and a StepOne Plus ${ }^{\mathrm{TM}}$ Real Time PCR system (Applied Biosystems; Thermo Fisher Scientific Inc.). Optimal reagent concentration and reaction condition described in the manufacturer's instructions were followed. The thermocycling conditions used for qPCR were as follows: $50^{\circ} \mathrm{C}$ for $2 \mathrm{~min}$, $95^{\circ} \mathrm{C}$ for $20 \mathrm{sec}$; followed by 40 cycles of denaturation at $95^{\circ} \mathrm{C}$ for $1 \mathrm{sec}$ and annealing/extension at $60^{\circ} \mathrm{C}$ for $20 \mathrm{sec}$. The $2^{-\Delta \Delta \mathrm{Cq}}$ method was used to determine gene expression levels (23). RT-qPCR reactions of undetermined $\mathrm{Cq}$ were assigned $\mathrm{Cq}=36$ cycle. GAPDH was used as a quantitative normalization reference. Primer sequences of the TaqMan Gene Expression assays are available in the following IDs: Glyceraldehyde 3-phosphate dehydrogenase (GAPDH; Cf04419463), collagen type VII alpha 1 chain (COL7A1; Cf02690281), AKT serine/threonine kinase 3 (AKT3; Cf02704523), ERBB receptor feedback inhibitor 1 (ERRFI1; Cf02653684), inhibitor of nuclear factor kappa B kinase subunit beta (IKBKB; Cf02695869), nerve growth factor (NGF; Cf02697134), epidermal growth factor receptor (EGFR; CF02626541), matrix metalloproteinase 9 (MMP9; CF02621845) and interleukin (IL)6 (Cf02624282). The details of the mentioned IDs can be found in the following website: https://www.thermofisher.com/order/genome-database/.

Statistical analysis. GraphPad Prism 7 (www.graphpad. com) was used for statistical analysis. Hierarchical clustering analysis was performed on $\log _{10}$ ratio with every gene expression from each sample. Hierarchical clustering was done with Euclidean distance metrics and complete linkage algorithm. Comparisons between the group (healthy, $n=12$; melanoma, $\mathrm{n}=17$ ) of the RT-qPCR data were performed using the Mann-Whitney U-test. A P-value $<0.05$ was considered to indicate a statistically significant difference.

\section{Results}

RNA-seq. RNA-seq was performed successfully for 11 samples (healthy controls, 3; melanoma, 8). Sequences were submitted to SRA databases (PRJNA527141). All sequence data were $2 \times 100 \mathrm{bp}$ in length with high quality metrics ( $>36$ Phred score). The total number of read pairs ranged from 44 million to 47 million. Approximately $83 \%$ (range: $81-84 \%$ ) of the read pairs were mapped to gene track (Canfam3.1). The percentage of genomic mapping was similar between the control and melanoma samples (means \pm SD: $83.743 \pm 0.357$ and $83.023 \pm 0.645 \%$, respectively) (Fig. 1A), suggesting that no significant biases were introduced during data generation between the groups $(\mathrm{P}=0.133)$. Mapping statistics indicated that the data were of high quality and uniform (no outliers regarding the genome). Principal component analysis of the expressed genes revealed a clear separation of the control group from the melanoma group (Fig. 1B). The status of the top 20 expressed genes in the healthy group was compared with the expression in the melanoma group. In total, 11 of the top 20 expressed genes in the healthy group were not observed in the melanoma group (Fig. 1C). KRT13 was the most highly expressed gene in the controls and MT-ATP6 was the most highly expressed gene in the melanoma group (Table SII). Known melanoma oncogenes, such as COL1A1, Vimentin and SPARC, were among the top 10 expressed genes in the melanoma group, while these genes were absent in the healthy group. These results revealed that the data had sufficient sequencing depth and were suitable for further differential expression analysis.

Identification and characterization of DEGs. To identify DEGs in the melanoma samples, we set up the following stringent criteria: FDR $<0.05, \mathrm{FC}>2$, and maximum group mean $>5$ (RPKM). This criterion identified 2,555 DEGs (Fig. 2A), including 1,421 upregulated and 1,134 downregulated genes (Tables SIII and SIV). The magnitude of the FC was higher in the downregulated group. In addition, 364 DEGs annotated by Ensembl were defined as novel genes, as they did not match species-specific entries in the UniProtKB/Swiss-Prot or 


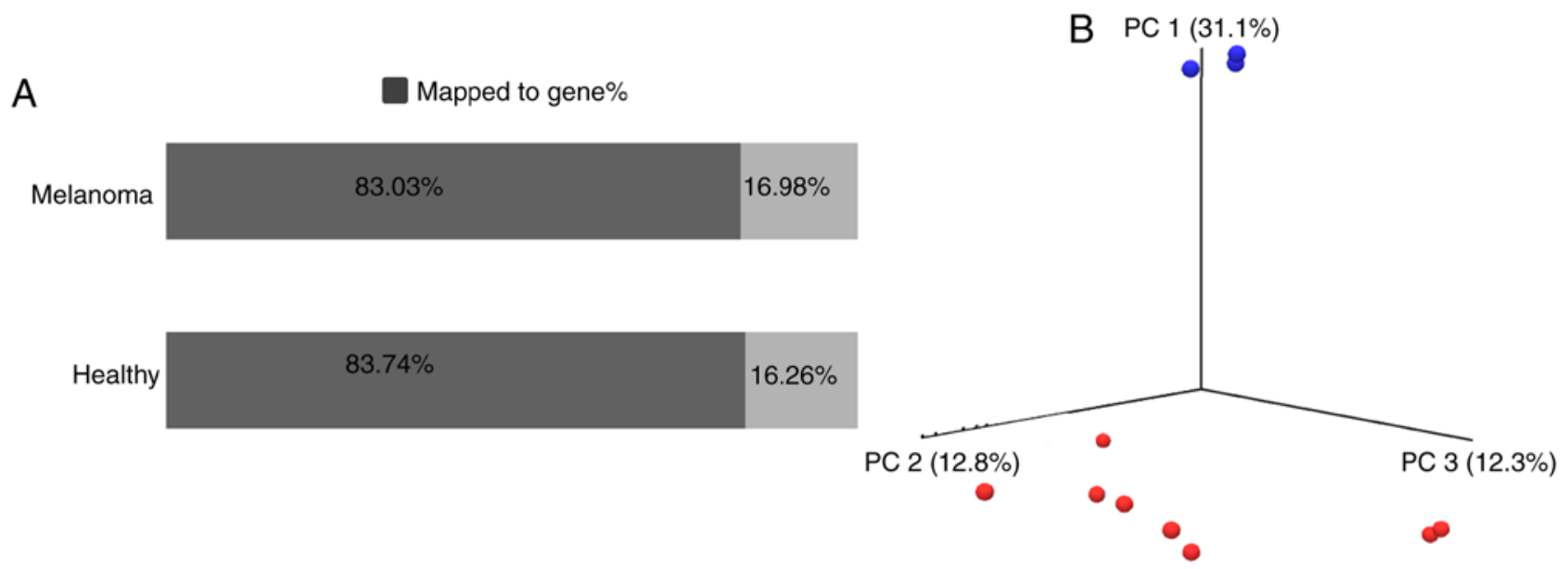

C Gene name/Ensembl ld KRT13

MT-ATP6

MT-CO1

KRT71

COX3

ENSCAFG00000023728

MT-CO2

$\underline{S 100 A 8}$

ENSCAFG00000029470

MT-ATP8

ND4

RPL13A

KRT15

MT-ND1

$\underline{\text { RPL17 }}$

TPT1

RPS6

S100A6

ENSCAFG00000018586

MT-CYB

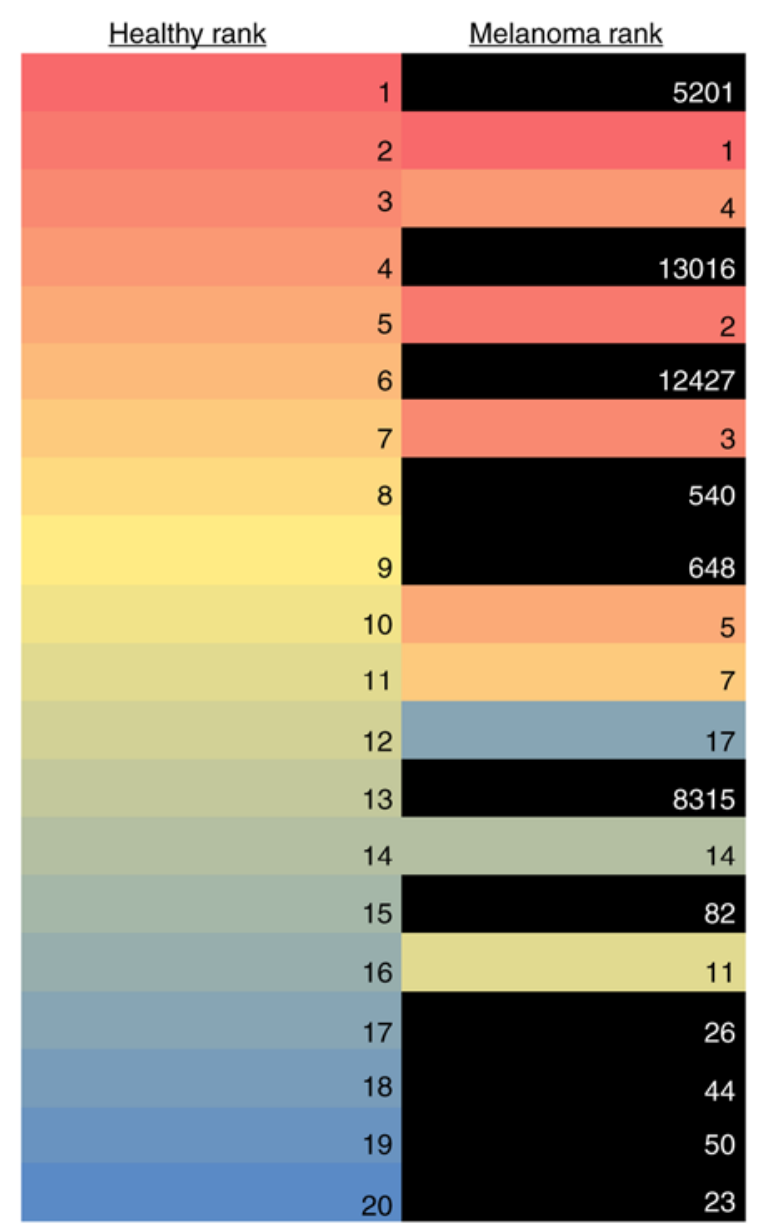

Figure 1. Reads characterization of RNA-seq from canine oral melanoma. (A) Mapped percentage of the reads against the reference genome. Percentages of the mapped reads were estimated with the average percentage of mapped reads from each group. Healthy, $n=3$; melanoma, $n=8$. (B) Principal component analysis of variance from transformed RNA seq reads counts for whole transcriptome by CLC Workbench. Axis indicates the variance contribution. Blue is for healthy and red is for melanoma samples. (C) Comparison of top twenty expressed genes in healthy ( $\mathrm{n}=3$ ) and melanoma ( $\mathrm{n}=8$ ) group. Number and color gradient (red to blue) were used to indicate highest to lowest ranking. Uncommon genes between the groups are underlined and positions are marked black color in melanoma. PC, principal component.

RefSeq databases; these genes included 219 upregulated and 145 downregulated genes (Tables I and SV).

We then classified the DEGs based on expression according to a previous study, with slight modifications (24). Genes were defined as very rare (5-15 RPKM), rare (16-99 RPKM), moderately abundant (100-499 RPKM) and abundant ( $>500$ RPKM). The majority of genes were categorized as very rare $(44.8 \%)$ and rare $(45.5 \%)$, followed by moderately abundant (7.7\%) and abundant (2.0\%) (Fig. 2B). Similarly, the novel genes were mostly categorized as very rare (45.32\%) and rare $(39.56 \%)$, followed by moderately abundant $(12.91 \%)$ and abundant (2.2\%) (Fig. 2C).

We then examined the 'on-off' genes in melanoma. Genes that were highly expressed ( $>5$ RPKM maximum group 

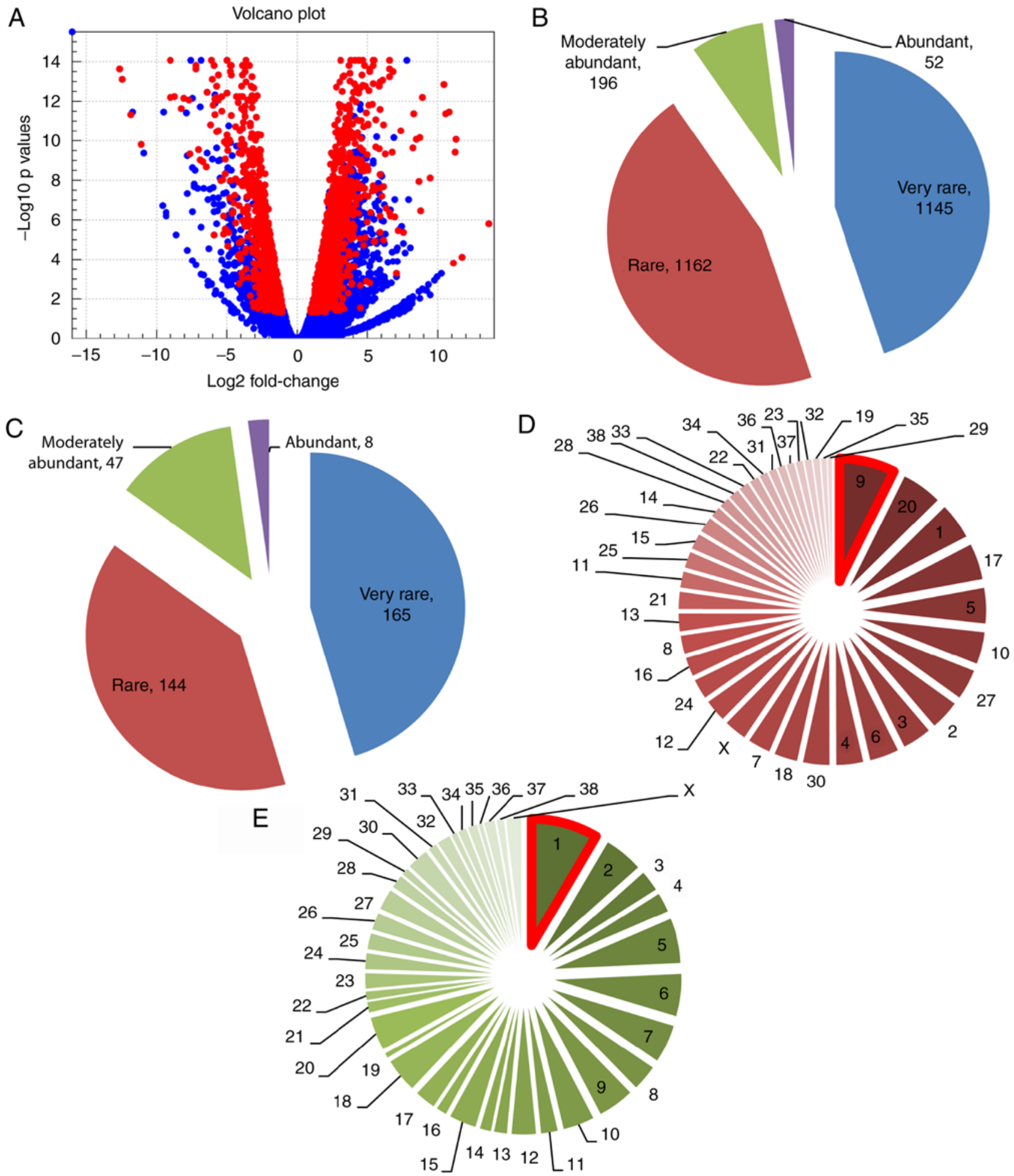

Figure 2. Differentially expressed genes from RNA-seq and their chromosomal location. (A) Volcano plot representing the differential expression of genes from RNA-seq. Each dot indicates one gene. A red dot indicates significant genes according to our stringent criterion, (FC) $>2$ and maximum group mean $>5$ (RPKM). The $\mathrm{x}$-axis indicates the $\log 2$ fold change of the genes comparing healthy and melanoma group; the $y$-axis indicates the - $\log _{10}$ false discovery rate (FDR). (B and C) Overall expression abundance of known and novel differentially expressed genes respectively. Numbers indicate the number of genes in each category: Very rare (5-15 RPKM), rare (16-99 RPKM), moderately abundant (100-499 RPKM) and abundant (>500 RPKM). (D and E) Chromosomal locations of differentially expressed genes. Numbers indicate the corresponding chromosome identity. The chromosome with the highest number of differentially expressed genes is indicated by the red border; (D) upregulated and (E) downregulated genes in the chromosomal locations.

mean) in one group with no expression in the other group ( $<1$ RPKM min group mean) and FDR as ' 0 ' were defined as 'on-off' genes. We identified 321 'on-off' genes, including 80 'on' (upregulated) genes and 241 'off' (downregulated) genes (Tables SVI and SVII). Among the 'on' genes, $B G N$, $C X C L 8$ and $P I 3$ were abundant genes ( $>500$ RPKM), whereas 14 'off' genes, including 3 keratin genes (KRT13, KRT71 and $K R T 78$ ), were abundant. In the novel gene group, we identified 

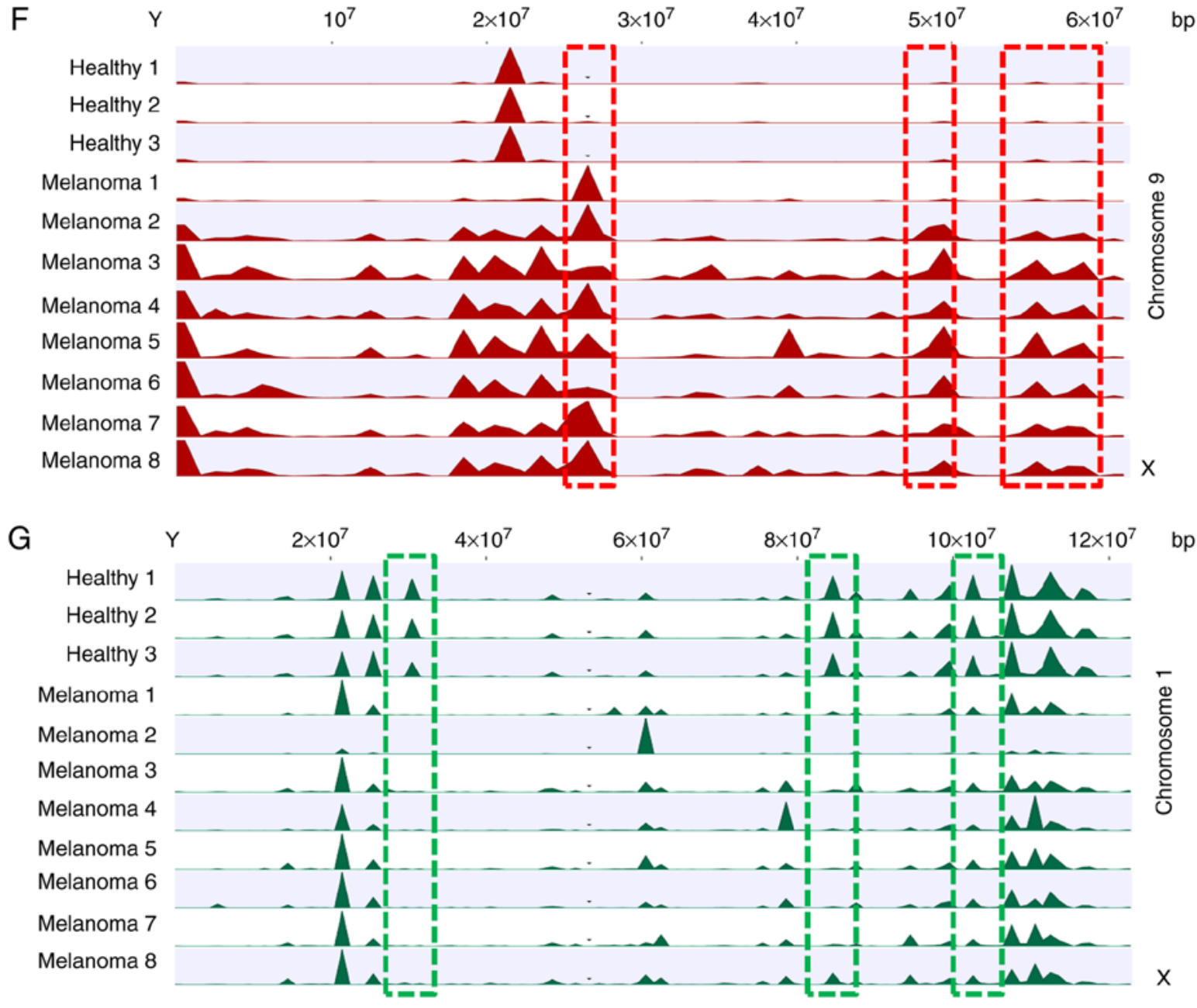

Figure 2. Continued. Differentially expressed genes from RNA-seq and their chromosomal location. (F and G) Reads mapped from each individual sample to the CFA1 and CFA9 regions from RNA-seq. Dotted lines indicated the mapped sequence variation of every sample between the groups. The X is the length of the chromosome and $\mathrm{Y}$ is the mapped sequences of each sample.

48 'on-off' genes (13 'on' and 35 'off' genes). Two genes were abundant (>500 RPKM) in each group (Tables SVIII and SIX). The abundant 'on-off' genes are presented in Table II.

To identify which chromosome harbored the majority of the DEGs, we analyzed the chromosomal location of all DEGs. We found that the highest number of upregulated genes $(n=104)$ were on $\mathrm{CFA}_{9}$ (dog chromosome 9) and the highest number of downregulated genes $(n=96)$ were on $\mathrm{CFA}_{1}$ (dog chromosome 1) (Fig. 2D and E). We also observed that 12 upregulated and 13 downregulated novel genes were located on $\mathrm{CFA}_{9}$ and $\mathrm{CFA}_{1}$, respectively (Table SV). Of note, the highest numbers of 'on' genes $(n=8)$ and 'off' genes $(n=26)$ were on $\mathrm{CFA}_{9}$ and $\mathrm{CFA}_{1}$, respectively (Tables SVI and SVII). When sequence reads were mapped against these 2 chromosomes, there were missing peaks or new peaks (peaks were made by the mapped sequence in the region) in each group (Fig. 2F and G).

We then performed functional analysis of the DEGs. Using the PANTHER classification system (25) DEGs produced 1,701 protein hits with 24 protein classes (Fig. S2A and B). The most abundant group of genes was in hydrolase $(8.70 \%)$. Relatively higher percentages of upregulated genes were in the signaling molecule, enzyme modulator, receptor, extracellular matrix protein, defense/immunity protein and cell adhesion molecule.
Immuno-related genes are also investigated by comparing the immune-genes from ImmPort resources (26). We found 174 and 75 immunogenes in the up- and downregulated group, respectively. In both groups, antimicrobial-related immunogens were abundant (Fig. S2C). Subsequently, we performed overrepresentation enrichment analysis (ORA) and found chemokines and antimicrobials were 2 significant $(\mathrm{P}<0.05)$ terms in the upregulated group with the highest enrichment ratio (chemokines, 1.6; antimicrobials, 1.3), respectively. The term chemokines was most enriched in the downregulated group, but did not bear statistical significance (data not shown).

\section{$G O$, pathway and transcription factor analysis}

$G O$ analysis. We then analyzed the DEGs by WebGestalt using the GSEA method. GO analysis categorizes DEGs into 3 categories: i) Biological process (BP); ii) cellular component (CC); and iii) molecular function (MF). In total 18 GO terms were significantly enriched (Fig. 3A). Defense response (GO: 0006952), cell-cell signaling (GO: 0007267), extracellular matrix (GO: 0031012), collagen trimer (GO: 0005581), cytokine receptor binding (GO: 0005126) and cytokine activity (GO: 0005125) were the top enriched terms in each category. Other significant GO terms (gliogenesis, taxis, 
Table I. Top 20 novel differentially expressed genes in canine oral melanoma.

\begin{tabular}{|c|c|c|c|c|c|}
\hline Ensembl ID & Chromosome & Region & $\begin{array}{l}\text { Max group } \\
\text { mean }\end{array}$ & $\begin{array}{l}\log _{2} \text { fold } \\
\text { change }\end{array}$ & $\begin{array}{c}\text { FDR } \\
\text { P-value }\end{array}$ \\
\hline ENSCAFG00000023728 & 17 & $61715810 . .61716667$ & 6965.176802 & -13.0728668 & 0 \\
\hline ENSCAFG00000029470 & 7 & $\begin{array}{l}\text { Complement } \\
(43565980 . .43569673)\end{array}$ & 4441.530653 & -6.317147271 & $8.66135 \mathrm{E}-15$ \\
\hline ENSCAFG00000018586 & 4 & $67701614 . .67703002$ & 1576.83413 & -1.476555989 & 0.044341327 \\
\hline ENSCAFG00000032057 & 26 & $27624214 . .27624534$ & 1030.146501 & 6.625580707 & $3.22992 \mathrm{E}-14$ \\
\hline ENSCAFG00000031806 & 26 & $27626671 . .27632004$ & 645.9357198 & 7.033040174 & 0 \\
\hline ENSCAFG00000030258 & 8 & $\begin{array}{l}\text { Complement } \\
(72906321 . .73387840)\end{array}$ & 585.378127 & 7.09342349 & 0 \\
\hline ENSCAFG00000017655 & 30 & $\begin{array}{l}\text { Complement } \\
(35713470 . .35737559)\end{array}$ & 554.1272685 & 1.5182161 & 0.02925338 \\
\hline ENSCAFG00000000471 & 12 & $742518 . .744376$ & 502.6434471 & -14.74161241 & 0 \\
\hline ENSCAFG00000031786 & 26 & $27605067 . .27616302$ & 497.5464419 & 7.498002132 & 0 \\
\hline ENSCAFG00000015206 & 21 & $40680858 . .40685074$ & 476.1739252 & 7.802839134 & 0 \\
\hline ENSCAFG00000030164 & $\mathrm{X}$ & $\begin{array}{l}\text { Complement } \\
(82986436 . .82986741)\end{array}$ & 473.8678307 & -1.319491428 & 0.025365105 \\
\hline ENSCAFG00000019812 & 6 & $\begin{array}{l}\text { Complement } \\
(42202578 . .42207944)\end{array}$ & 360.4657394 & -2.977400921 & 4.13027E-06 \\
\hline ENSCAFG00000023111 & 17 & $\begin{array}{l}\text { Complement } \\
(60984425 . .60987378)\end{array}$ & 354.3255735 & -1.423676 & 0.00159774 \\
\hline ENSCAFG00000016966 & 30 & $27636259 . .27664073$ & 309.6540468 & 2.174448832 & 0.018722413 \\
\hline ENSCAFG00000032259 & 9 & $\begin{array}{l}\text { Complement } \\
(37617977 . .37622560)\end{array}$ & 298.0600534 & -8.627843471 & 0 \\
\hline ENSCAFG00000019141 & $\mathrm{X}$ & $\begin{array}{l}\text { Complement } \\
(119204969 . .119205259)\end{array}$ & 277.0573496 & -1.266862717 & 0.016078337 \\
\hline ENSCAFG00000032358 & 8 & $\begin{array}{l}\text { Complement } \\
(72847361 . .72852219)\end{array}$ & 268.1075815 & 5.707864264 & 5.27351E-11 \\
\hline ENSCAFG00000029493 & 26 & $27620223 . .27620543$ & 264.9025566 & 6.875187743 & $1.58428 \mathrm{E}-08$ \\
\hline ENSCAFG00000014627 & 3 & $60899870 . .60901258$ & 261.1790712 & 8.044118048 & 1.11703E-08 \\
\hline ENSCAFG00000012022 & 17 & $59698670 . .59701290$ & 254.5146118 & -4.447052200 & $8.66135 \mathrm{E}-15$ \\
\hline
\end{tabular}

FDR, false discovery rate.

immune response, growth factor activity, glycosaminoglycan binding, G-protein coupled receptor binding) related with the altered physiology during melanoma progression. Among the 18 significant GO terms, 9 were directly related to cytokines. Taken together, the GO results indicate that most DEGs are involved in cytokine-oriented functions.

Pathway analysis. We performed pathway analysis by 2 methods: GAGE and GSEA. In total, 9 common pathways were significantly enriched in both methods (Fig. 3B). To rank the pathways, the position of each analysis was taken and the average was examined. Cytokine-cytokine receptor interaction (CFA04060), focal adhesion (CFA04510) and ECM-receptor interaction (CFA04512) were the top 3 pathways. PI3K-AKT (CFA04151) and TNF (CFA04668) signaling pathways were also present in our analysis.

Enriched transcription factor motif. To examine motifs up to $4 \mathrm{~kb}$ around the transcription start sites of the DEGs, we used GSEA within WebGestalt. In total, 10 transcription site binding motifs were significantly enriched in the upregulated DEGs (Fig. 3C). Among these 10, 6 were known and 4 were unknown motifs that do not match any known transcription factor binding site from the database (v7.4 TRANSFAC). The binding motifs for ATF1 and NF- $\kappa \mathrm{B}$ were observed in the highest number of upregulated DEGs.

Cross species analysis of human and dog melanoma. We analyzed 2 human melanoma RNA-seq from GEO datasets (please see Materials and methods). To evaluate the pattern of FC of the dog DEGs in human melanoma, we converted the genes to the human orthologues and compared the FC with the human melanoma study without considering statistical significance. The analysis of the human melanoma tissue results revealed that $63 \%$ of the upregulated genes and $40 \%$ of the downregulated genes had the same direction of FC between the species (Fig. 4A). In the case of human melanoma cell lines, we observed 58 and $47 \%$ similarities in FC, respectively 
Table II. Abundant 'on-off' genes in canine oral melanoma.

\begin{tabular}{|c|c|c|c|c|}
\hline Name & Chromosome & Max group mean & $\log _{2}$ fold change & FDR P-value \\
\hline BGN & $\mathrm{X}$ & 750.6354384 & 5.44424503 & 0 \\
\hline CXCL8 & 13 & 718.7157383 & 8.318634019 & 0 \\
\hline PI3 & 24 & 625.5995841 & 8.475895656 & 0 \\
\hline KRT13 & 9 & 19890.23609 & -11.27810332 & 0 \\
\hline KRT71 & 27 & 7541.688327 & -15.13319019 & 0 \\
\hline S100A8 & 7 & 5616.157022 & -6.39785469 & 0 \\
\hline ARSF & $\mathrm{X}$ & 1426.603766 & -12.19831506 & 0 \\
\hline TGM3 & 24 & 1376.03872 & -15.43829609 & 0 \\
\hline AQP3 & 11 & 1324.472821 & -11.7832697 & 0 \\
\hline S100A14 & 7 & 1165.913739 & -9.555767692 & 0 \\
\hline SPRR3 & 17 & 1090.426813 & -13.23899417 & 0 \\
\hline S100A2 & 7 & 1023.838115 & -8.469980831 & 0 \\
\hline SFN & 2 & 769.2134926 & -8.549424168 & 0 \\
\hline RHCG & 3 & 723.8378326 & -12.84073049 & 0 \\
\hline SPINK5 & 2 & 646.388121 & -11.37480897 & 0 \\
\hline S100A16 & 7 & 549.0286762 & -6.490015324 & 0 \\
\hline KRT78 & 27 & 508.5240706 & -11.77812871 & 0 \\
\hline ENSCAFG00000031806 & 26 & 645.936 & 7.03304 & 0 \\
\hline ENSCAFG00000030258 & 8 & 585.378 & 7.09342 & 0 \\
\hline ENSCAFG00000023728 & 17 & 6965.18 & -13.073 & 0 \\
\hline ENSCAFG00000000471 & 12 & 502.643 & -14.742 & 0 \\
\hline
\end{tabular}

FDR, false discovery rate.

(Fig. S3A). Of note, when we compared the statistically significant genes between the species (FDR $<0.05$, FC $\geq 2$; common DEGs), the percentage of shared upregulated genes increased (tissue, 88\%; cell line, 62\%) in both experiments (Figs. 4B and S3B, and Tables SX-SXIII). These findings indicate a marked overlap in upregulated genes or oncogenes between human and dog melanoma.

To further understand the association between human and dog tissue melanoma, we performed hierarchical clustering analysis. Common DEGs between the 2 melanoma (human and dog) tissue experiments were selected and expression values were considered from all other experiments for clustering. Clustering analysis of dog and human melanoma tissues, cell line and prostate cancer revealed that dog melanoma clustered together with a subset of human tissue melanoma samples (Fig. 4C). These results indicate the closer transcriptomic similarities between dog and human melanoma compared with other types of cancer. Prostate cancer data were included to indicate the dissimilarities in different types of cancer between the species.

We found that 429 upregulated melanoma signature genes, including 105 genes commonly upregulated in all 3 melanoma sets, 284 genes upregulated in human and dogs tissue melanoma, and 40 genes upregulated in cell line and dog melanoma, were the main causative driver genes for melanoma development (Table SXIV). Approximately half $(n=41,51 \%)$ of the on genes identified in dog melanoma samples were present in this group.

To examine the processes of melanoma development in the 2 species, we performed GSEA of common DEGs from
3 experiments. In total 10 pathways had an FDR $<0.06$ and 3 had a normalized enrichment score $>2$ (Fig. 4D). The top 3 pathways were immune and signaling related pathways. The leading edge genes of these pathways were also deregulated in a similar pattern in both species (Fig. 4D, lower panel).

We established a network from common DEGs by STRING and performed analysis by MCODE in Cytoscape. Twelve cluster networks were obtained (Fig. 4E). The majority of the genes of the first 3 networks encode signaling peptides. Genes in the first network are collagen and integrin genes (Fig. 4F; upper left panel). The second and third cluster genes are genes encoding cytokines-chemokines and growth factors (Fig. 4F; upper right and lower panels). As the FC of genes in the network was the same between the species, this indicated that these genes may exhibit similar melanoma promoting networking function between the species.

Validation of DEGs by RT-qPCR. To confirm the result of RNA-seq we validated several genes by RT-qPCR. We confirmed that COL7A1, AKT3, ERRFI1, IKBKB, NGF, IL6, $M M P 9$ and $E G F R$ genes were differentially expressed in dog melanoma (Fig. 4G). Similar fold changes of the genes were observed between RNA-seq and RT-qPCR.

\section{Discussion}

To the best of our knowledge, this is the first report of comprehensive RNA-seq in canine oral malignant melanoma. A previous study performed RNA-seq on canine cutaneous melanoma (27). 
A

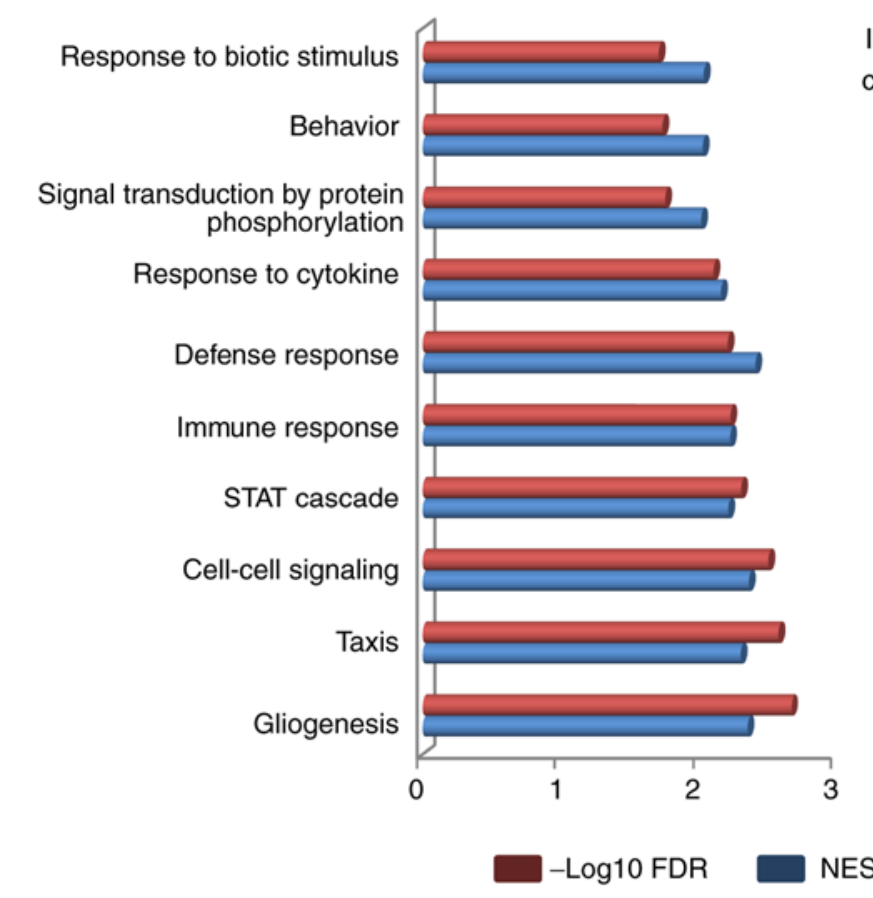

B

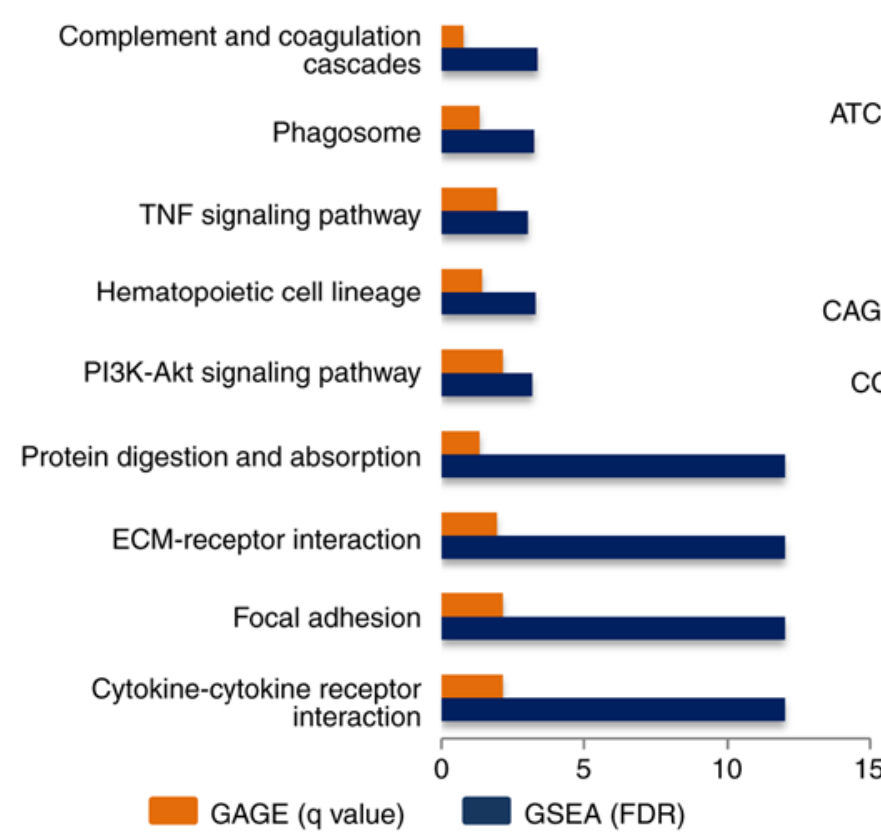

Cellular component

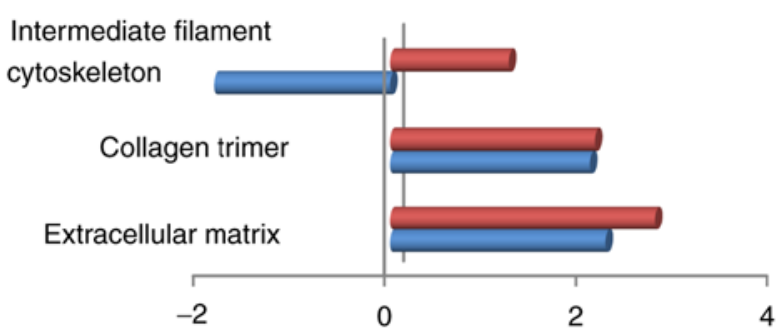

Molecular function

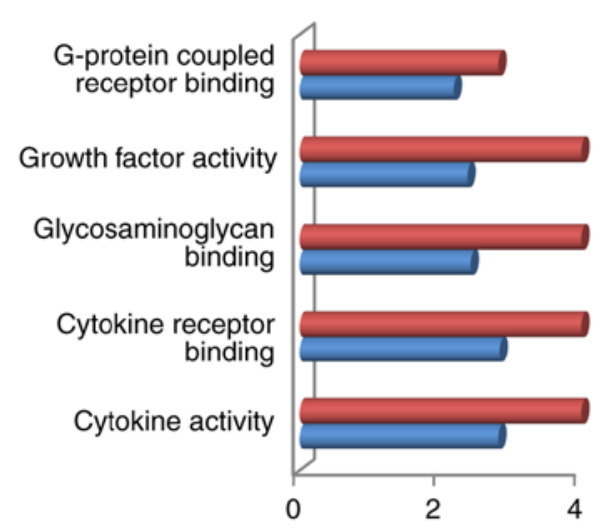

TF GSEA analysis

ALX4

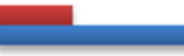

ATCMNTCCGY_unknown motif

HTF

NFSF

AGNWMCNNNGAC_unknown

motif

CCANNAGRKGGC_unknown motif

PBX

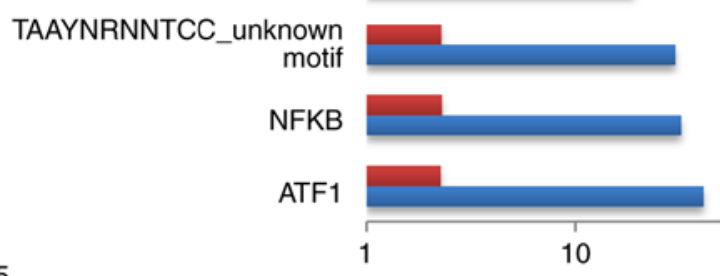

NES $\square$ Leading edge genes count

Figure 3. Gene Ontology, pathway and transcription factor (TF) analysis of the differentially expressed genes. (A) Gene Ontology analysis of significant terms in biological process (BP), cellular component (CC) and molecular function (MF). Blue bars indicate the normalized enrichment score (NES); red bars indicate the - $\log$ false discovery rate (FDR). (B) Pathways that were significant between two methods are shown. The x-axes represent the -log value of generally applicable gene set enrichment (GAGE) q value and gene set enrichment analysis (GSEA) FDR. (C) Enriched TFs are shown with NES and number of leading edge genes in $\log$ scale at the $\mathrm{x}$-axis.

Oral melanoma is the most frequent site for malignant melanoma compared with cutaneous type $(11,28)$. In addition, previous studies have demonstrated that oral melanoma in dogs can be used as a model for human melanoma $(2,10,11)$.

The results of this study revealed that COL1A1, SPARC and VIM were the top highly expressed DEGs in canine oral malignant melanoma. These genes have also been well studied in human melanoma or other types of cancer for their oncogenic behavior (29-32). In comparison, KRT13, KRT71 and $S 100 A 8$ were not expressed in the melanoma group. In a study on human squamous cell carcinomas of the head and neck and esophagus, KRT13 was found to be epigenetically silenced, while the chromosomal location of the S100A8 gene was found to be frequently altered or deleted and downregulated $(33,34)$. 
A

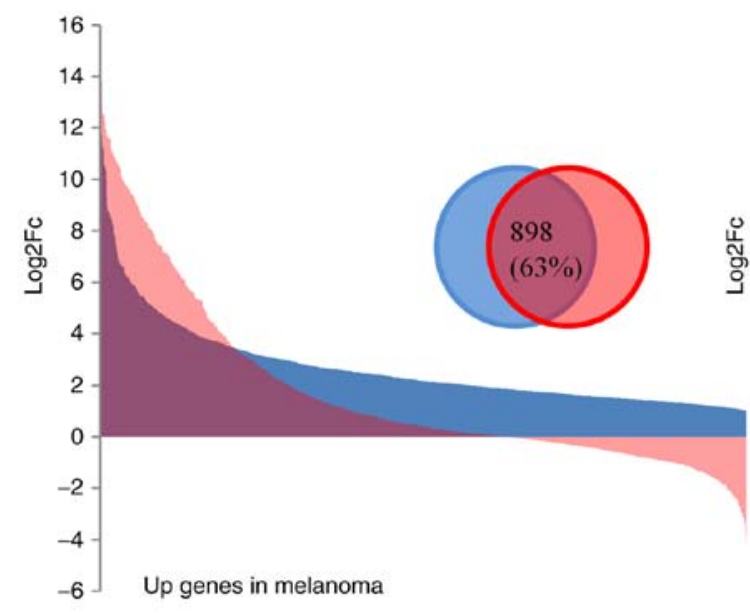

B

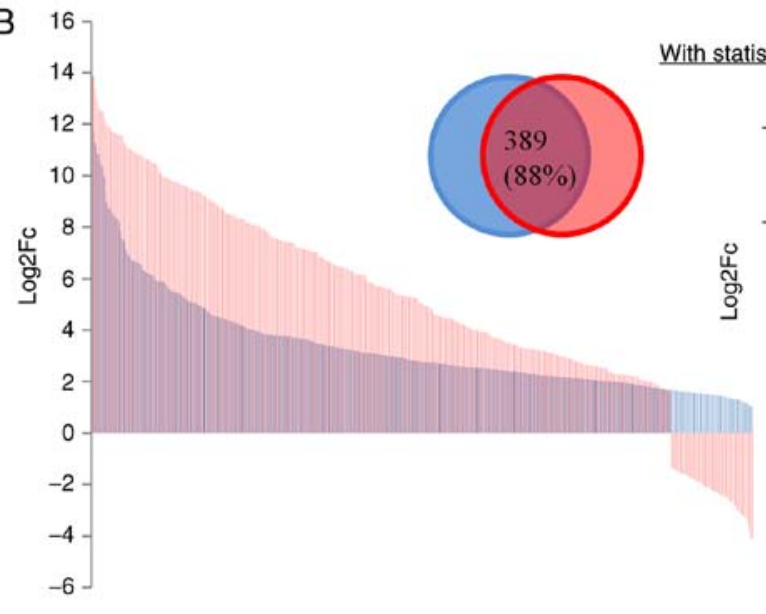

Up genes in melanoma
Without statistical significance

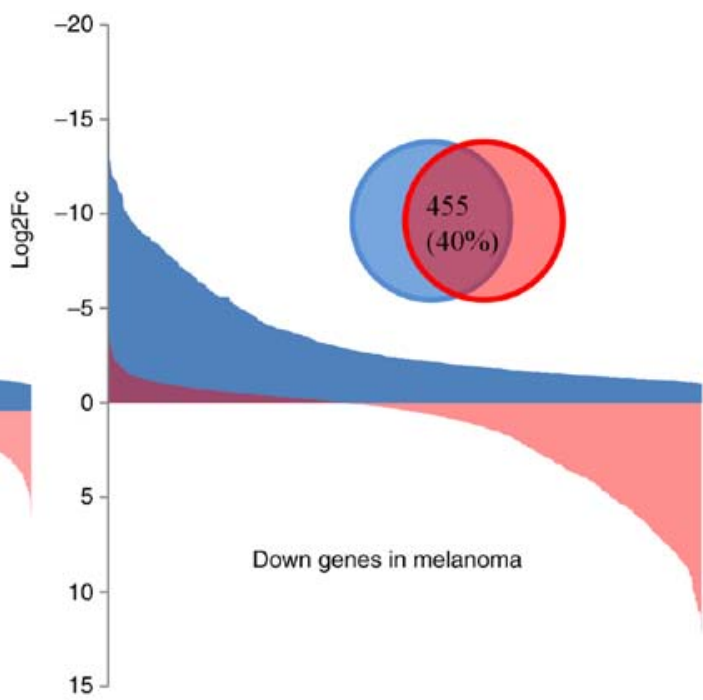

With statistical significance

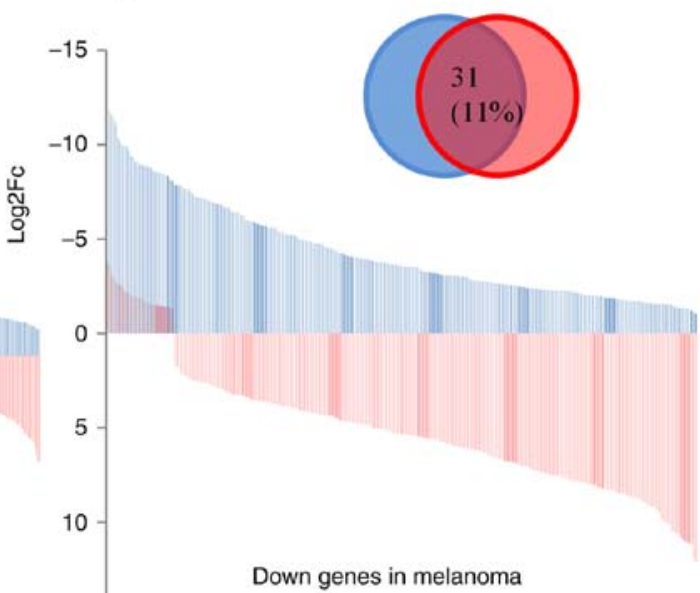

15

C

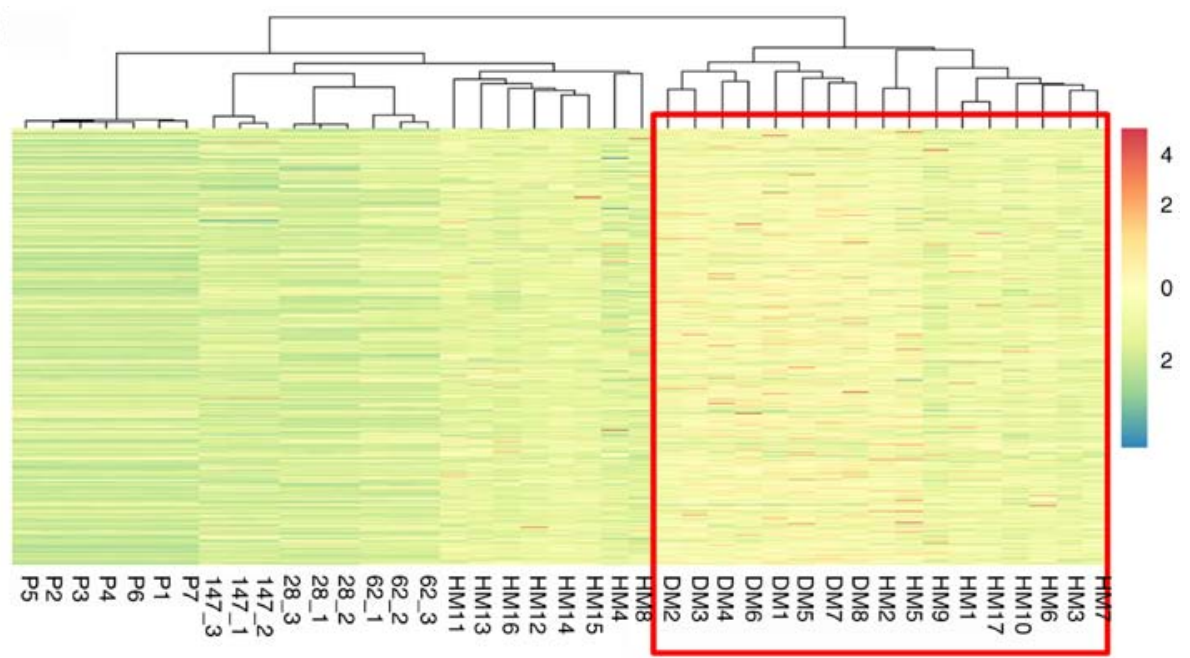

Figure 4. Differentially expressed genes between human and dog melanoma. (A and B) Gene fold change (FC) between the species with or without considering statistical significance in dog melanoma. Numbers and percentages of common up- and downregulated genes are shown in the overlapping region. The $\mathrm{x}$-axis is the number of genes and the y-axis indicates the FC. (C) Heatmap with cluster analysis showing the expression of common oncogenes between prostate cancer cell lines (LNCaP-P1-P7), human melanoma cell lines [SK_MEL_28 (28_1-3), SK_MEL_147 (147_1-3), UACC_62 (62_1-3)], canine oral melanoma (DM_1-8) and human tissue melanoma (HM_1-17). The color gradient on the right indicates the expression values. Euclidean hierarchical clustering with complete linkage was used. Dog and human clustered together is indicated within the red line. 

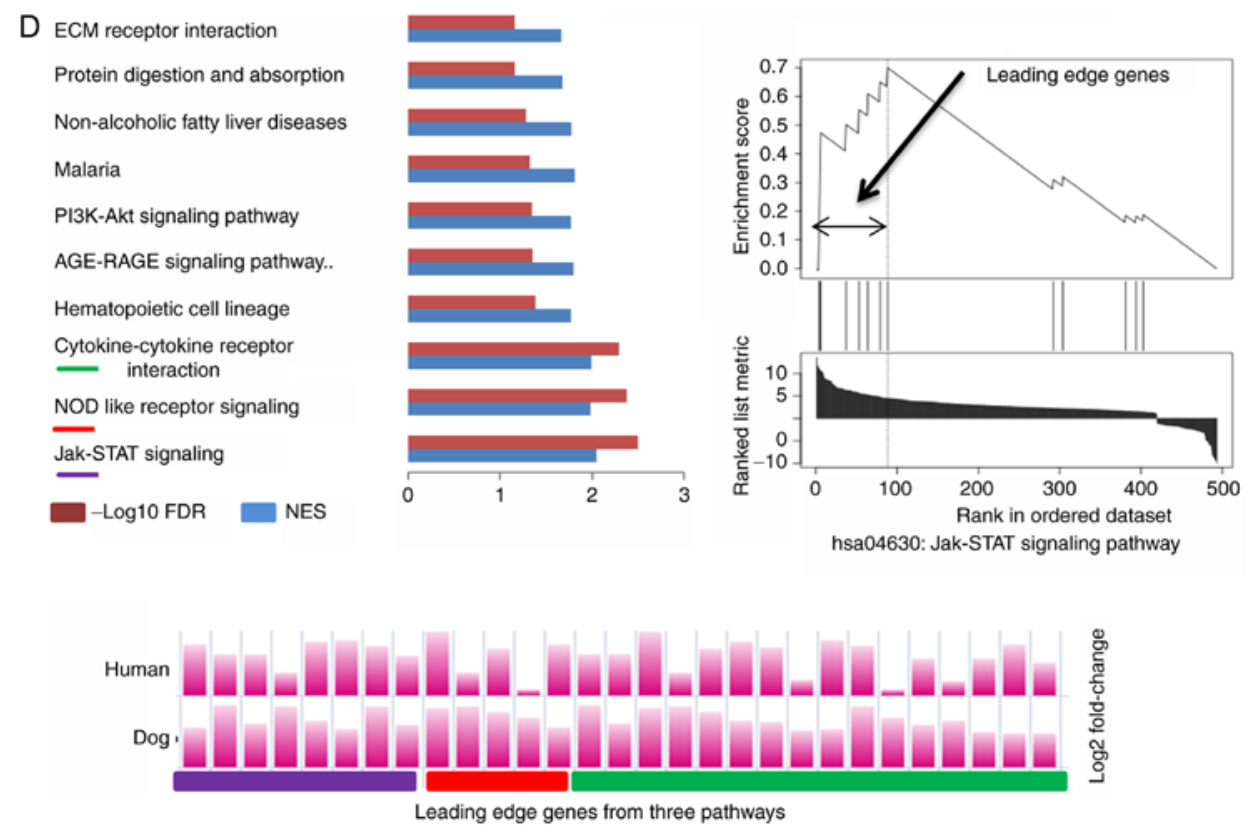

E
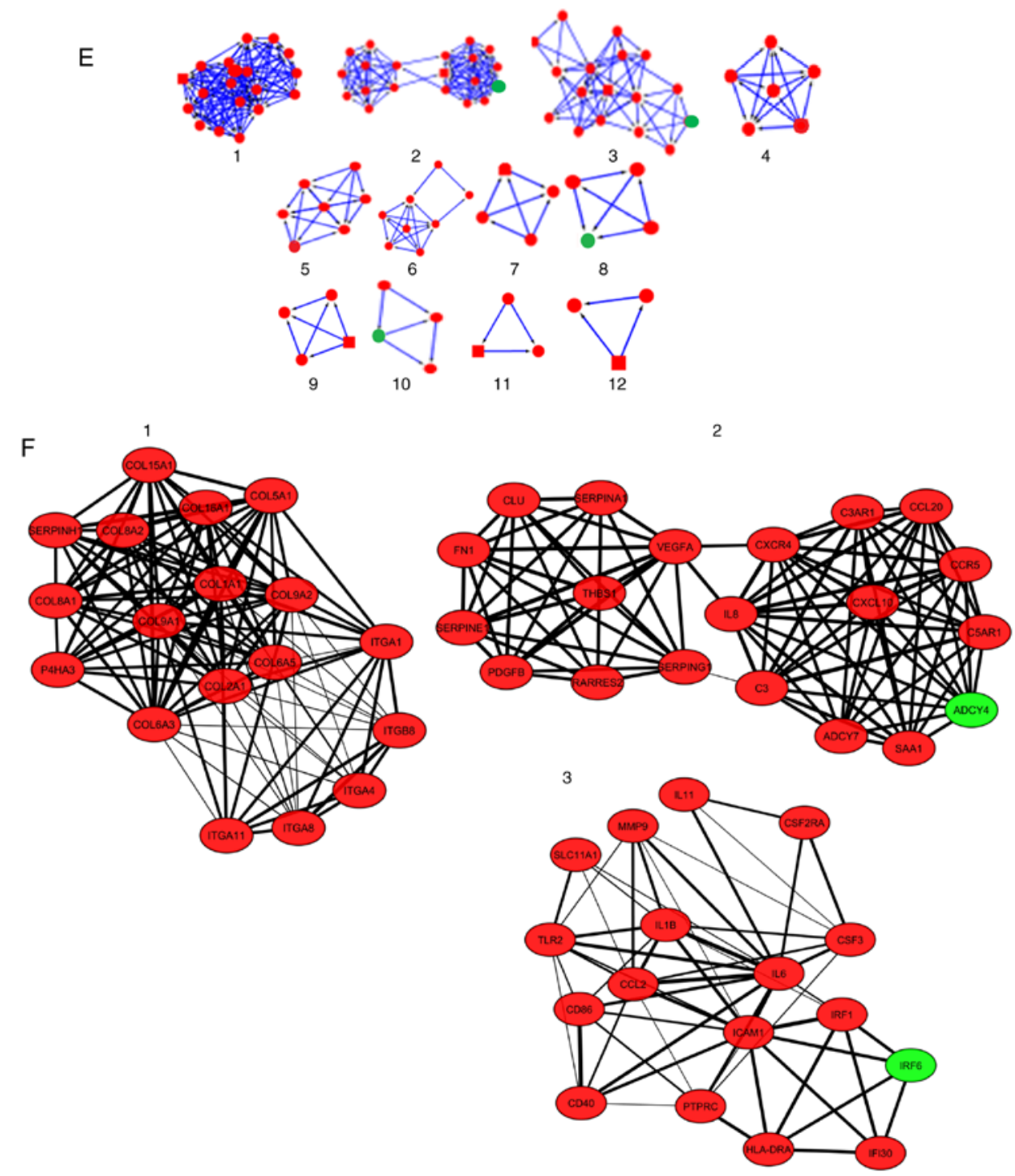

Figure 4. Continued. Differentially expressed genes between human and dog melanoma. (D) Common enriched pathways between humans and dogs. Schematic on top right panel indicates how leading edge genes were defined. Fold changes of the leading edge genes from the top 3 pathways in both species are shown on the bottom panel. FDR, false discovery rate; NES, normalized enrichment score. (E) Schematic presentation of 12 network clusters established by the common differentially expressed genes. (F) The first three clusters are shown in which a node indicates a gene and the lines between them indicate the edge. Red color indicates upregulated genes and green represents downregulated genes. 

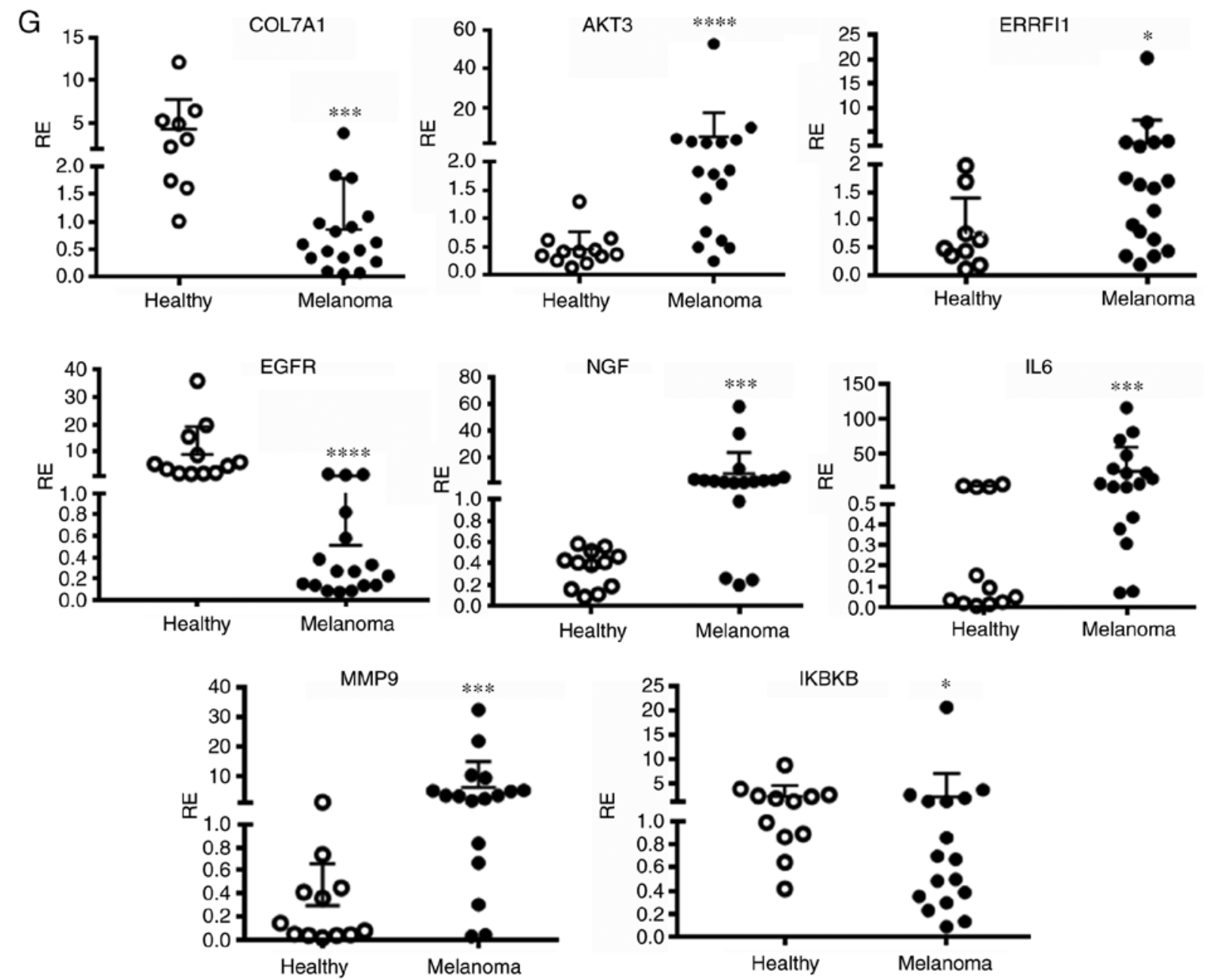

Figure 4. Continued. Differentially expressed genes between human and dog melanoma. (G) Relative expression of COL7A1 (healthy control, n=9), AKT3, ERRFI1, EGFR, NGF, IL6, MMP9 and IKBKB genes examined by RT-qPCR in healthy oral tissue $(\mathrm{n}=12)$ and oral melanoma $(\mathrm{n}=17)$. ${ }^{*} \mathrm{P}<0.05$, ${ }^{* * *} \mathrm{P}<0.01$, ${ }^{* * * * * *} \mathrm{P}<0.0001$. The bars indicate standard deviation (SD). RE, relative expression; COL7A1, collagen type VII alpha 1 chain; AKT3, AKT serine/threonine kinase 3; ERRFI1, ERBB receptor feedback inhibitor 1; EGFR, epidermal growth factor receptor; NGF, nerve growth factor; IL6, interleukin 6; MMP9, matrix metallopeptidase 9; IKBKB, inhibitor of nuclear factor kappa B kinase subunit beta.

However, genes that are expressed in either of the group bear more significance than those with less magnitude of change. These genes bear more importance for biomarker or therapeutic study. We thus found the 80 genes that were expressed only in canine malignant melanoma ( $>5$ RPKM maximum group mean) compared with healthy tissue $(<1$ RPKM min group mean), with the aim of identifying genes that were turned on during melanoma progression. Using this criterion, $B G N, C X C L 8$ and $P I 3$ were identified as 3 abundant genes in canine malignant melanoma. Only $C X C L 8$ was previously investigated in dogs to be increased in hemangiosarcoma (35). $B G N, C X C L 8$ and PI3 have previously been studied in human melanoma and other types of cancer (36-38). The abundant genes are only approximately $2 \%$ of the total DEGs. As highly expressed genes (abundant) are transcribed upon the essential demands of cells, their exact association with and involvement in melanoma progression warrants further investigation.

Cytogenic analysis of the DEGs revealed that $\mathrm{CFA}_{1}$ harbored the majority of the 'off' genes or downregulated genes. Loss of alleles or abnormalities in $\mathrm{HSA}_{1}$ (human chromosome 1) in human malignant melanoma was previously reported $(39,40)$. This suggests that chromosome 1 is important in melanoma and the function in melanoma suppression is conserved in both species. We examined the distribution of DEGs in 24 protein classes and found the highest number of genes within the hydrolase category (220 genes). Most of the hydrolases were proteases (135 genes). Proteases are involved in regulatory signaling networks with kinases or other factors can function to transmit oncogenic signals in the tumor micro-environment. The Protein classification of these DEGs provides an important foundation for further understanding of the pathogenesis of melanoma. Melanoma is one of the most immunogenic cancers. The immunogenic landscape of dog oral melanoma DEGs revealed the enrichment of chemokines and antimicrobials genes. Previous studies have proven that chemokines play specific roles in human melanoma tumor growth and metastasis $(41,42)$. Chemokine-based therapy is also under continuous investigation (43). Moreover, antimicrobial immunogenes may enrich as a first line defense of the cancer cells, although many of them can regulate chemokines and other immunogenic signals.

GO analysis revealed that the majority of proteins encoded by DEGs were distributed in the extracellular 
domain or cytoplasm. We hypothesized that these proteins drive cells to undergo several physiological processes to generate the oncogenic microenvironment. In this study, different response, cytokine and signaling process-related genes were enriched and were involved in G-protein, growth factor, glycosaminoglycan and cytokine-related activity. G-protein-coupled receptors are key players in the regulation of various pathophysiological responses to initiate cancer development, including melanoma. GPCR-targeted drugs have exhibited excellent therapeutic benefits in human cancers (44). Another significant term, growth factor activity involved in cancer, was first discovered in the 1950s by Cohen et al (45). Subsequent studies demonstrated various roles of growth factors in the tumor microenvironment including in melanoma (46). Glycosaminoglycans and the conjugated proteins were reported to be involved in the tumor micro-environment and often perform crucial functions along with cytokine and growth factors (47).

In this study, we identified 9 pathways enriched in the DEGs using 2 methods to avoid possible bias. ECM receptor interaction, focal adhesion, protein digestion and absorption and cytokine receptor interaction were the most enriched pathways and along with 3 PI3K-AKT signaling pathways were previously reported to be involved in canine cutaneous melanoma (27). Pathway analysis has been useful for the analysis of experimental high-throughput biological data to facilitate data interpretation. For example, $I K K \beta$, one of the major positive regulators of the NF- $\mathrm{KB}$ transcription factor, was found to be downregulated in canine oral melanoma (Fig. S4). However, several target genes of NF-kB were upregulated, indicating that NF- $\mathrm{KB}$ was activated. We also examined the transcription factors binding motifs that may represent the transcription factors of upregulated genes and found that the NF- $\mathrm{KB}$ binding motif was the most enriched. This suggests that NF- $\kappa \mathrm{B}$ genes are activated through $\mathrm{NF}-\kappa \mathrm{B}-\mathrm{independent} \mathrm{mechanisms} \mathrm{or} \mathrm{that} \mathrm{NF- \kappa B} \mathrm{is} \mathrm{activated}$ through IKK $\beta$-independent mechanisms. However, when we analyzed the pathways for the common deregulated genes between humans and dogs, we found that JAK-STAT was the most enriched pathway. Among the target genes of $\mathrm{NF}-\kappa \mathrm{B}, S T A T 3, N F-\kappa B 1$ and $R E L A$ share the highest number of genes (http://www.grnpedia.org/trrust/result.php?gene= STAT3\&species=human\&confirm $=$ ). This indicates that these target genes can be transactivated by either NF- $\mathrm{BB}$ or STAT3, or both factors. In this study, we found that 39 targets were upregulated in the dog melanoma data and 21 were significant. In the human data, among the 21 orthologues, 17 were upregulated (Table SXV). These data again suggested that one or both of the transcription factors may be activated. As $I K K \beta$ was downregulated, we hypothesized that the canonical pathway was not activated in dogs. The target genes can be transcribed by either non-canonical or atypical pathways of NF- $\mathrm{kB}$ or by STAT3. A previous study also demonstrated that feedback loops exist between both signaling pathways. IL6, as one of the targets of NF- $\kappa B$, can be regulated by STAT3 activation (48). IL6 was expressed in both human and dog melanoma. One study demonstrated that the pro-survival function of NF- $\mathrm{BB}$ was related to its functional interaction with the PI3K/AKT/mTOR signaling pathway. AKT engages mainly with IKK $\alpha$ instead of IKK $\beta$ in promoting NF- $\kappa \mathrm{B}$ activation (49). NGF can activate NF- $\kappa \mathrm{B}$ by the atypical pathway (50). Phosphorylation mediates the activation of STAT3 through TrkA by NGF (51). Therefore, IL6 may be a crucial regulator in melanoma initiation by regulating the STAT3/NF- $\mathrm{kB}$ loop, while the atypical NF-kB pathway is maintained in dogs by NGF. Further studies are required to examine the potential for IL6 and NGF as novel therapeutic targets in melanoma for both species. RT-qPCR analysis confirmed the upregulation of $N G F, A K T$, and ILG and IKK $\beta$ downregulation in canine melanoma tissue samples.

Several studies have demonstrated clinicopathological and molecular similarities in melanoma between dogs and humans $(10,11,52)$. However, to the best of our knowledge, no study to date has revealed the oncogenic transcriptomic similarities of melanoma between these species. In this study, we evaluated the common DEGs between the species. Among the upregulated genes in dog melanoma, 88 and $62 \%$ orthologous genes were also upregulated in human melanoma tissue and cell lines, respectively. In addition, among the 429 upregulated melanoma signature genes, 48 were previously reported in melanoma according to the Melanoma Gene Database (MGDB) (53) (Table SXIV). This result indicates that oncogenic functions of these genes for melanoma progression are conserved between the two species. Previous studies have also demonstrated that higher homology of known cancer genes, as well as mutation or inactivation events in cancer or other diseases are shared between these 2 species $(11,54,55)$. The findings of this study further support the similarities in melanoma progression between dogs and humans. Several subtypes of melanoma have been identified in humans (9). Dog oral melanoma has been suggested as a model for human mucosal and the triple wild-type subtype $(10,11)$. The cluster analysis of this study with melanoma and prostate cancer revealed that dog melanoma clustered with a group of human tissue melanoma. These results again affirm previous studies that a human melanoma subtype is similar to dog melanoma. This study also demonstrates that the dog model will be more efficient to investigate or develop novel therapeutics compared with cell lines.

We also created a protein network using a human database. We speculated that deregulated proteins/genes in melanoma interact to drive disease progression. Functional association in melanoma has been found from the protein interaction network (56). In this study, each network cluster contained genes that perform similar functions, such as the first cluster that mostly contained collagen and integrins mainly involved with extracellular matrix-related functions. Our results suggest that the same network exists in both species, as the genes show the same trend of expression in melanoma. The roles of collagen and integrins in cancer have been well studied $(57,58)$. Potential therapeutic targets can be attained from this type of interaction network strategy, which is also reported by a previous study (56).

In conclusion, this study successfully identified the transcriptomic aberrations in canine oral melanoma. Our evidence demonstrating the similarity of melanoma between the 2 species further emphasizes dogs as a suitable pre-clinical model for human melanoma. By comparing the melanoma transcriptome between the 2 species, we identified the key 
genes and molecular pathways for further study to develop more effective therapeutic approaches to melanoma.

\section{Acknowledgements}

The authors would like to thank Ms. Ayako Masuda, Veterinary Teaching Hospital, Joint Faculty of Veterinary Medicine, Kagoshima University; for assisting with the experiments. The authors would also like to thank Edanz Group (www.edanzediting.com/ac) for editing a draft of this manuscript.

\section{Funding}

This study was supported by the Japan Society for the Promotion of Science KAKENHI (grant nos. 17H03926, 15H14872, 25292180 and 22780283).

\section{Availability of data and materials}

The datasets used and/or analyzed during the current study are available from the corresponding author on reasonable request.

\section{Authors' contributions}

NaM, MMR and YCL were involved in the conception and design of the study. NaM, MMR, AAH and HWC developed the methodology. NaM, MMR, YCL, AAH, HWC, YT, HK, HH NoM, TN and RF were involved in data acquisition. NaM, MMR, YCL, AAH and HWC analyzed and interpreted the data. The manuscript was written by MMR and critically reviewed by all other authors. Administrative and technical support was provided by NaM, YT, HK, HH, NoM, TN and RF. Overall the study was supervised by NaM. All authors have read and approved the final manuscript.

\section{Ethics approval and consent to participate}

Informed consent to use the specimens in this study was obtained from the owners of the dogs from which tissues were obtained. The animal experiments were approved by the Kagoshima University's Laboratory Animal Committee.

\section{Patient consent for publication}

Not applicable.

\section{Competing interests}

The authors state that there are no competing interests.

\section{References}

1. Rowell JL, McCarthy DO and Alvarez CE: Dog models of naturally occurring cancer. Trends Mol Med 17: 380-388, 2011.

2. Simpson RM, Bastian BC, Michael HT, Webster JD, Prasad ML, Conway CM, Prieto VM, Gary JM, Goldschmidt MH, Esplin DG, et al: Sporadic naturally occurring melanoma in dogs as a preclinical model for human melanoma. Pigment Cell Melanoma Res 27: 37-47, 2014.

3. Siegel RL, Miller KD and Jemal A: Cancer statistics, 2018. CA Cancer J Clin 68: 7-30, 2018.
4. Ossio R, Roldán-Marín R, Martínez-Said H, Adams DJ and Robles-Espinoza CD: Melanoma: A global perspective. Nat Rev Cancer 17: 393-394, 2017.

5. Kong Y, Si L, Zhu Y, Xu X, Corless CL, Flaherty KT, Li L, Li H, Sheng X, Cui C, et al: Large-scale analysis of KIT aberrations in Chinese patients with melanoma. Clin Cancer Res 17: 1684-1691, 2011.

6. Smith SH, Goldschmidt MH and McManus PM: A comparative review of melanocytic neoplasms. Vet Pathol 39: 651-678, 2002.

7. Treggiari E, Grant JP and North SM: A retrospective review of outcome and survival following surgery and adjuvant xenogeneic DNA vaccination in 32 dogs with oral malignant melanoma. J Vet Med Sci 78: 845-850, 2016.

8. Prouteau A and André C: Canine melanomas as models for human melanomas: Clinical, histological, and genetic comparison. Genes (Basel) 10: pii: E501, 2019.

9. Cancer Genome Atlas Network: Genomic classification of cutaneous melanoma. Cell 161: 1681-1696, 2015.

10. Hernandez B, Adissu HA, Wei BR, Michael HT, Merlino G and Simpson RM: Naturally occurring canine melanoma as a predictive comparative oncology model for human mucosal and other triple wild-type melanomas. Int J Mol Sci 19: pii: E394, 2018.

11. Gillard M,Cadieu E, De Brito C, Abadie J, Vergier B, Devauchelle P, Degorce F, Dréano S, Primot A, Dorso L, et al: Naturally occurring melanomas in dogs as models for non-UV pathways of human melanomas. Pigment Cell Melanoma Res 27: 90-102, 2014.

12. Mochizuki H, Kennedy K, Shapiro SG and Breen MB: BRAF mutations in canine cancers. PLoS One 10: e0129534, 2015.

13. Chu PY, Pan SL, Liu CH, Lee J, Yeh LS and Liao AT: KIT gene exon 11 mutations in canine malignant melanoma. Vet J 196: 226-230, 2013.

14. Lindblad-Toh K, Wade CM, Mikkelsen TS, Karlsson EK, Jaffe DB, Kamal M, Clamp M, Chang JL, Kulbokas EJ III, Zody MC, et al: Genome sequence, comparative analysis and haplotype structure of the domestic dog. Nature 438: 803-819, 2005.

15. Bednarski R, Grimm K, Harvey R, Lukasik VM, Penn WS, Sargent B and Spelts K; American Animal Hospital Association: AAHA anesthesia guidelines for dogs and cats. J Am Anim Hosp Assoc 47: 377-385, 2011.

16. Hoeppner MP, Lundquist A, Pirun M, Meadows JR, Zamani N, Johnson J, Sundström G, Cook A, FitzGerald MG, Swofford R, et al: An improved canine genome and a comprehensive catalogue of coding genes and non-coding transcripts. PLoS One 9: e91172, 2014.

17. Mortazavi A, Williams BA, McCue K, Schaeffer L and Wold B: Mapping and quantifying mammalian transcriptomes by RNA-Seq. Nat Methods 5: 621-628, 2008.

18. Kinsella RJ, Kähäri A, Haider S, Zamora J, Proctor G, Spudich G, Almeida-King J, Staines D, Derwent P, Kerhornou A, et al: Ensembl BioMarts: A hub for data retrieval across taxonomic space. Database (Oxford) 2011: bar030, 2011.

19. Wang J, Vasaikar S, Shi Z, Greer M and Zhang B: WebGestalt 2017: A more comprehensive, powerful, flexible and interactive gene set enrichment analysis toolkit. Nucleic Acids Res 45 (W1): W130-W137, 2017.

20. Luo W, Pant G, Bhavnasi YK, Blanchard SG Jr and Brouwer C: Pathview Web: User friendly pathway visualization and data integration. Nucleic Acids Res 45 (W1): W501-W508, 2017.

21. Szklarczyk D, Franceschini A, Wyder S, Forslund K, Heller D, Huerta-Cepas J, Simonovic M, Roth A, Santos A, Tsafou KP, et al: STRING v10: Protein-protein interaction networks, integrated over the tree of life. Nucleic Acids Res 43 (Database Issue): D447-D452, 2015.

22. Lopes CT, Franz M, Kazi F, Donaldson SL, Morris Q and Bader GD: Cytoscape Web: An interactive web-based network browser. Bioinformatics 26: 2347-2348, 2010.

23. Livak KJ and Schmittgen TD: Analysis of relative gene expression data using real-time quantitative PCR and the 2(-Delta Delta C(T)) method. Methods 25: 402-408, 2001.

24. Li RW and Schroeder SG: Cytoskeleton remodeling and alterations in smooth muscle contractility in the bovine jejunum during nematode infection. Funct Integr Genomics 12: 35-44, 2012.

25. Mi H, Muruganujan A, Casagrande JT and Thomas PD: Large-scale gene function analysis with the PANTHER classification system. Nat Protoc 8: 1551-1566, 2013.

26. Bhattacharya S, Dunn P, Thomas CG, Smith B, Schaefer H, Chen J, Hu Z, Zalocusky KA, Shankar RD, Shen-Orr SS, et al: ImmPort, toward repurposing of open access immunological assay data for translational and clinical research. Sci Data 5: $180015,2018$. 
27. Brachelente C, Cappelli K, Capomaccio S, Porcellato I, Silvestri S, Bongiovanni L, De Maria R, Verini Supplizi A, Mechelli L and Sforna M: Transcriptome analysis of canine cutaneous melanoma and melanocytoma reveals a modulation of genes regulating extracellular matrix metabolism and cell cycle. Sci Rep 7: 6386, 2017.

28. Spangler WL and Kass PH: The histologic and epidemiologic bases for prognostic considerations in canine melanocytic neoplasia. Vet Pathol 43: 136-149, 2006.

29. Miskolczi Z, Smith MP, Rowling EJ, Ferguson J, Barriuso J and Wellbrock C: Collagen abundance controls melanoma phenotypes through lineage-specific microenvironment sensing. Oncogene 37: 3166-3182, 2018.

30. Han MJ, Wang H, Beer LA, Tang HY, Herlyn M and Speicher DW: A systems biology analysis of metastatic melanoma using in-depth three-dimensional protein profiling. Proteomics 10 : 4450-4462, 2010.

31. Tichet M, Prod'Homm V, Fenouille N, Ambrosetti D, Mallavialle A, Cerezo M, Ohanna M, Audebert S, Rocchi S, Giacchero D, et al: Tumour-derived SPARC drives vascular permeability and extravasation through endothelial VCAM1 signalling to promote metastasis. Nat Commun 6: 6993, 2015.

32. Wurth L, Papasaikas P, Olmeda D, Bley N, Calvo GT, Guerrero S, Cerezo-Wallis D, Martinez-Useros J, García-Fernández M, Hüttelmaier S, et al: UNR/CSDE1 drives a post-transcriptional program to promote melanoma invasion and metastasis. Cancer Cell 30: 694-707, 2016.

33. Naganuma K, Hatta M, Ikebe T and Yamazaki J: Epigenetic alterations of the keratin 13 gene in oral squamous cell carcinoma. BMC Cancer 14: 988, 2014

34. Khammanivong A, Sorenson BS, Ross KF, Dickerson EB, Hasina R, Lingen MW and Herzberg MC: Involvement of calprotectin (S100A8/A9) in molecular pathways associated with HNSCC. Oncotarget 7: 14029-14047, 2016.

35. Kim JH, Frantz AM, Anderson KL, Graef AJ, Scott MC Robinson S, Sharkey LC, O'Brien TD, Dickerson EB and Modiano JF: Interleukin-8 promotes canine hemangiosarcoma growth by regulating the tumor microenvironment. Exp Cell Res 323: 155-164, 2014.

36. Andrlová H, Mastroianni J, Madl J, Kern JS, Melchinger W Dierbach H, Wernet F, Follo M, Technau-Hafsi K, Has C, et al: Biglycan expression in the melanoma microenvironment promotes invasiveness via increased tissue stiffness inducing integrin- $\beta 1$ expression. Oncotarget 8: 42901-42916, 2017.

37. Nagarsheth N, Wicha MS and Zou W: Chemokines in the cancer microenvironment and their relevance in cancer immunotherapy. Nat Rev Immunol 17: 559-572, 2017.

38. Labidi-Galy SI, Clauss A, Ng V, Duraisamy S, Elias KM, Piao HY, Bilal E, Davidowitz RA, Lu Y, Badalian-Very G, et al: Elafin drives poor outcome in high-grade serous ovarian cancers and basal-like breast tumors. Oncogene 34: 299-309, 2015.

39. Smedley D, Sidhar S, Birdsall S, Bennett D, Herlyn M, Cooper C and Shipley J: Characterization of chromosome 1 abnormalities in malignant melanomas. Genes Chromosom Cancer 28: 121-125, 2000.

40. Dracopoli NC, Harnett P, Bale SJ, Stanger BZ, Tucker MA, Housman DE and Kefford RF: Loss of alleles from the distal short arm of chromosome 1 occurs late in melanoma tumor progression. Proc Natl Acad Sci USA 86: 4614-4618, 1989.

41. Kuo PT, Zeng Z, Salim N, Mattarollo S, Wells JW and Leggatt GR: The role of CXCR3 and its chemokine ligands in skin disease and cancer. Front Med (Lausanne) 5: 271, 2018.
42. Payne AS and Cornelius LA: The role of chemokines in melanoma tumor growth and metastasis. J Invest Dermatol 118 915-922, 2002.

43. Jacquelot N, Duong CPM, Belz GT and Zitvogel L: Targeting chemokines and chemokine receptors in melanoma and other cancers. Front Immunol 9: 2480, 2018.

44. Lappano R and Maggiolini M: G protein-coupled receptors: Novel targets for drug discovery in cancer. Nat Rev Drug Discov 10: 47-60, 2011.

45. Cohen S, Levi-Montalcini R and Hamburger V: A nerve growth-stimulating factor isolated from sarcom as 37 and 180 . Proc Natl Acad Sci USA 40: 1014-1018, 1954.

46. Witsch E, Sela M and Yarden Y: Roles for growth factors in cancer progression. Physiology (Bethesda) 25: 85-101, 2010.

47. Afratis N, Gialeli C, Nikitovic D, Tsegenidis T, Karousou E, Theocharis AD, Pavão MS, Tzanakakis GN and Karamanos NK: Glycosaminoglycans: Key players in cancer cell biology and treatment. FEBS J 279: 1177-1197, 2012.

48. Wang Y, van van Boxel-Dezaire AH, Cheon $\mathrm{H}$, Yang $\mathrm{J}$ and Stark GR: STAT3 activation in response to IL- 6 is prolonged by the binding of IL-6 receptor to EGF receptor. Proc Natl Acad Sci USA 110: 16975-16980, 2013.

49. Dan HC, Cooper MJ, Cogswell PC, Duncan JA, Ting JP and Baldwin AS: Akt-dependent regulation of NF- $\{$ kappa\}B is controlled by mTOR and Raptor in association with IKK. Genes Dev 22: 1490-1500, 2008.

50. Gentry JJ, Casaccia-Bonnefil P and Carter BD: Nerve growth factor activation of nuclear factor kappaB through its p75 receptor is an anti-apoptotic signal in RN22 schwannoma cells. J Biol Chem 275: 7558-7565, 2000

51. Ng YP, Cheung ZH and Ip NY: STAT3 as a downstream mediator of Trk signaling and functions. J Biol Chem 281: 15636-15644, 2006.

52. Hendricks WPD, Zismann V, Sivaprakasam K, Legendre C, Poorman K, Tembe W, Perdigones N, Kiefer J, Liang W, DeLuca V, et al: Somatic inactivating PTPRJ mutations and dysregulated pathways identified in canine malignant melanoma by integrated comparative genomic analysis. PLoS Genet 14: e1007589, 2018.

53. Zhang D, Zhu R, Zhang $\mathrm{H}$, Zheng $\mathrm{CH}$ and Xia J: MGDB: A comprehensive database of genes involved in melanoma. Database (Oxford) 2015: pii: bav097, 2015.

54. Bushell KR, Kim Y, Chan FC, Ben-Neriah S, Jenks A, Alcaide M, Fornika D, Grande BM, Arthur S, Gascoyne RD, et al: Genetic inactivation of TRAF3 in canine and human B-cell lymphoma. Blood 125: 999-1005, 2015.

55. Ulvé R, Rault M, Bahin M, Lagoutte L, Abadie J, De Brito C, Coindre JM, Botherel N, Rousseau A, Wucher V, et al: Discovery of human-similar gene fusions in canine cancers. Cancer Res 77: 5721-5727, 2017.

56. Li Z, Ivanov AA, Su R, Gonzalez-Pecchi V, Qi Q, Liu S, Webber P, McMillan E, Rusnak L, Pham C, et al: The OncoPPi network of cancer-focused protein-protein interactions to inform biological insights and therapeutic strategies. Nat Commun 8: 14356, 2017.

57. Hamidi $\mathrm{H}$, Pietilä $\mathrm{M}$ and Ivaska $\mathrm{J}$ : The complexity of integrins in cancer and new scopes for therapeutic targeting. Br J Cancer 115: 1017-1023, 2016

58. Chen P, Cescon M and Bonaldo P: Collagen VI in cancer and its biological mechanisms. Trends Mol Med 19: 410-417, 2013.

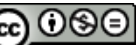

This work is licensed under a Creative Commons Attribution-NonCommercial-NoDerivatives 4.0 International (CC BY-NC-ND 4.0) License. 